\title{
Reação de cultivares de sorgo sacarino a Meloidogyne javanica, Rotylenchulus reniformis e Pratylenchus brachyurus
}

\author{
Cibelle da Silva Oliveira ${ }^{1}$, Guilherme Lafourcade Asmus ${ }^{2}$ \\ ${ }^{1}$ Universidade Estadual de Mato Grosso do Sul, UEMS, Unidade Universitária de Aquidauana, Aquidauana, MS, Brasil. E-mail: \\ cibelle_agronomia@hotmail.com \\ ${ }^{2}$ Empresa Brasileira de Pesquisa Agropecuária - Embrapa Agropecuária Oeste - CPAO, Dourados - MS, Brasil. E-mail: \\ guilherme.asmus@embrapa.br
}

Recebido: 18/05/2018; Aceito: 25/10/2018.

\section{RESUMO}

O objetivo do presente trabalho foi avaliar a reprodução de Meloidogyne javanica, Rotylenchulus reniformis e Pratylenchus brachyurus em raízes das cultivares de sorgo sacarino 'BRS 506', 'BRS 508', 'BRS 509' e 'BRS 511 ' em três experimentos conduzidos em casa de vegetação. As cultivares de sorgo foram semeadas em vasos de polietileno de $3 \mathrm{~L}$, preenchidos com substrato desinfestado, composto da mistura de solo e areia (2:1). Doze dias após a emergência, cada planta foi inoculada com populações puras de $M$. javanica, $R$. reniformis ou $P$. brachyurus, nos respectivos experimentos. Os padrões de susceptibilidade foram soja 'BRS 360RR' para $M$. javanica, algodão 'BRS Cedro' para R. reniformis e milho ‘BRS 1010' para P. brachyurus. Crotalária (Crotalaria spectabilis) foi utilizada como padrão de resistência em todos os experimentos. O delineamento experimental foi de blocos inteiramente casualizados com oito repetições. Aos 60 (M. javanica e $R$. reniformis) e 90 ( $P$. brachyurus) dias após a inoculação, os nematoides foram extraídos das raízes e estimados os fatores de reprodução (FR) e o número de nematoides por grama de raiz (NGR) das cultivares de sorgo. Com base nas variáveis avaliadas, as cultivares de sorgo sacarino 'BRS 506', 'BRS 508', 'BRS 509' e 'BRS 511' podem ser consideradas resistentes a $R$ reniformis e $M$. javanica, e suscetíveis a $P$. brachyurus.

Palavras-chave: Sorghum bicolor, nematoide das galhas, nematoide reniforme, nematoide das lesões radiculares.

\section{Reaction of sweet sorghum cultivars to Meloidogyne javanica, Rotylenchulus reniformis and Pratylenchus brachyurus}

\begin{abstract}
The objective of this work was to evaluate the reproduction of Meloidogyne javanica, Rotylenchulus reniformis and Pratylenchus brachyurus on roots of the sweet sorghum cultivars 'BRS 506', 'BRS 508', 'BRS 509', and 'BRS 511', in three greenhouse experiments. Sorghum seeds were sown in polyethylene pots of 3 L. filled with desinfested substrate, composed of a mixture of soil and sand (2:1). Twelve days after emergence, each plant was inoculated with pure populations of $M$. javanica, R. reniformis or P. brachyurus, respectively in each experiment. 'BRS 360RR' soybean, 'BRS Cedro' cotton and 'BRS 1010' corn cultivars were used as susceptible standards, respectively in $M$. javanica, $R$. reniformis or $P$. brachyurus experiments. (Crotalaria spectabilis) was used as resistant standard in all experiments. The experiments were conducted in a randomized block design with eight replications. After 60 or 90 days after the inoculations, respectively for $M$. javanica and $R$. reniformis or $P$. brachyurus experiments, nematodes were extracted from the roots and the reproduction factors and nematodes per gram of roots were estimated. Based on the evaluated variables, sweet sorghum cultivars 'BRS 506', 'BRS 508', 'BRS 509' and 'BRS 511' may be considered resistant to $R$ reniformis and $M$. javanica, and susceptible to $P$. brachyurus.
\end{abstract}

Keywords: Sorghum bicolor, root-knot nematode, reniforme nematode, root-lesions nematode. 


\section{Introdução}

O sorgo sacarino (Sorghum bicolor (L.) Moench) é uma importante alternativa para fornecimento de matéria-prima às destilarias, principalmente no período de entressafra da cana-de-açúcar, entre novembro e abril, em que há escassez de matéria prima para a produção de etanol e, assim, estabilizar a oferta de etanol combustível ao longo do ano no Brasil (Miranda, 2012). Do ponto de vista agronômico, o sorgo apresenta uma série de vantagens, tais como o estabelecimento da cultura por sementes, ciclo rápido e possibilidade de uso de mesma estrutura agroindustrial do setor sucroalcooleiro, o que pode contribuir para a viabilidade da adoção desta cultura, tanto na entressafra da cana-deaçúcar como na renovação de canaviais (May et al., 2015). Outra possibilidade é que o sorgo seja cultivado em sucessão ou rotação com culturas anuais, tais como soja [Glycine max (L.) Merrill], algodão (Gossypium hirsutum L.) ou milho (Zea mays L.).

A sequência de cultivo de plantas suscetíveis é um dos principais fatores para o aumento da população de fitonematoides em solos agrícolas (McSorley, 1998). Assim, ao ser inserido nos sistemas de produção em sucessão ou rotação com cana-de-açúcar, soja, algodão ou milho, o sorgo poderá favorecer o aumento da população de fitonematoides (Spaull e Cadet, 1990).

Os principais nematoides que ocorrem em cana-deaçúcar no Brasil são Meloidogyne javanica (Treub) Chitwood, M. incognita (Kofoid and White) Chitwood e Pratylenchus zeae Graham (Dinardo-Miranda et al., 2003). Em áreas de produção de soja, são mais frequentes as espécies $M$. javanica, M. incognita, Rotylenchulus reniformis Linford \& Oliveira, Heterodera glycines Ichinohe e, mais recentemente, $P$. brachyurus (Godfrey) Filipjev \& S. Stekhoven (Dias et al., 2010). M. incognita, $R$. reniformis e P. brachyurus também ocorrem e causam danos em algodoeiro (Galbieri et al., 2012) e $P$. zeae e $M$. incognita são comuns em milho (Lordello, 1981). À exceção de $H$. glycines, que apresenta alta especificidade à cultura da soja (Dias et al., 2010) e de $R$. reniformis (Asmus et al., 2008), todas as demais espécies supracitadas já foram relatadas parasitando a cultura do sorgo (Inomoto et al., 2008; Inomoto et al., 2006; Mcclure et al., 1999; Motalaote et al., 1987), para as quais se evidenciou variabilidade quanto à suscetibilidade.

Em virtude disso, ao definir-se um sistema de produção de sorgo sacarino em reforma de canavial ou em rotação ou sucessão a culturas para a produção de grãos ou fibras, torna-se fundamental o conhecimento da reação de cultivares aos principais nematoides que ocorrem na região. Nesse sentido, o presente trabalho teve como objetivo determinar a reação de quatro cultivares de sorgo sacarino: Meloidogyne javanica, Rotylenchulus reniformis e Pratylenchus brachyurus.

\section{Material e Métodos}

Foram conduzidos três experimentos em casa de vegetação, na Embrapa Agropecuária Oeste, em Dourados - MS, no período de abril a novembro de 2014. Como tratamentos, foram avaliados quatro cultivares de sorgo sacarino: 'BRS 506', 'BRS 508', 'BRS 509' e 'BRS 511', um padrão de suscetibilidade (variável, dependendo do nematoide) e crotalária (Crotalaria spectabilis L.) como padrão de resistência em todos os experimentos, totalizando seis tratamentos e oito repetições em delineamento experimental de blocos ao acaso.

O primeiro experimento foi conduzido nos meses de abril a julho, para avaliar a reação das cultivares de sorgo sacarino a $R$. reniformis. Como padrão de suscetibilidade ao nematoide, foi utilizado o algodoeiro da cultivar 'BRS Cedro'. O segundo foi realizado entre os meses de agosto a outubro para avaliar a reação a $M$. javanica, sendo padrão de suscetibilidade a soja "BRS 360RR", e o terceiro entre os meses de agosto a novembro, para avaliar a reação das mesmas cultivares a $P$. brachyurus, utilizando como padrão de suscetibilidade a cultura de milho da cultivar 'BRS $1010^{\prime}$.

A população de $M$. javanica foi obtida de raízes de soja e a espécie identificada pela análise da configuração perineal de 10 fêmeas (Taylor e Sasser, 1978). As populações de $R$. reniformis e P. brachyurus foram obtidas respectivamente de raízes de algodoeiro e de soja e das espécies identificadas por características morfométricas (Robinson et al., 1997; Gonzaga et al., 2016). Para a produção dos inóculos, M. javanica, $R$. reniformis e $P$. brachyurus foram multiplicados em raízes de tomateiro, maracujazeiro e quiabeiro, respectivamente, e extraídos das raízes pelo método de flotação centrífuga em solução de sacarose com adição de caulim, proposto por Coolen e D’Herde (1972), usando-se solução de hipoclorito de sódio comercial a $0,5 \%$ ao invés da água para triturar as raízes no liquidificador. Foram utilizados 5000 ovos/vaso de $M$. javanica, 1000 ovos/vaso de $R$. reniformis e 250 juvenis + adultos/vaso de $P$. brachyurus, respectivamente, nos experimentos 1, 2 e 3 .

As inoculações foram realizadas sempre aos dez dias após a semeadura, através da deposição de $5 \mathrm{~mL}$ da suspensão de inóculo, dividida em duas porções de 2,5 $\mathrm{mL}$ e em dois orifícios de $3,0 \mathrm{~cm}$ de profundidade no substrato, distantes $1,0 \mathrm{~cm}$ do colo das plantas.

As plantas foram irrigadas diariamente com volume de água suficiente para manter a umidade do solo próxima da capacidade de campo.

Aos 60 dias após a inoculação, foram realizadas as avaliações nos experimentos com $M$. javanica e $R$. reniformis. Devido ao comprovado desenvolvimento inicial mais lento de $P$. brachyurus e a consequente 
necessidade de tempo mais longo para atingir maior densidade populacional (Inomoto, 2011), as avaliações no experimento com esta espécie de nematoide foram realizadas 90 dias após a inoculação. As plantas foram seccionadas na altura do colo, descartando-se a parte aérea, e as raízes foram separadas do substrato, lavadas cuidadosamente em água corrente para retirada do excesso de substrato e colocadas sobre bancada forrada com papel absorvente, por 20 minutos, para retirada do excesso de água. Em seguida, as raízes foram pesadas e acondicionadas em sacos plásticos de $1,0 \mathrm{~kg}$, os quais foram mantidos sob refrigeração, a $8{ }^{\circ} \mathrm{C}$, até que se procedessem às extrações, utilizando-se os mesmos procedimentos empregados para obtenção do inóculo (Coolen e D'Herde, 1972). Todo o sistema radicular de cada planta foi utilizado para a extração dos nematoides.

Após a extração, os nematoides foram inativados em banho-maria a $52{ }^{\circ} \mathrm{C}$ por 5 minutos e fixados em formalina (2\%), ficando assim armazenados até serem quantificados. Para a quantificação, a suspensão contendo os nematoides e a formalina foi lavada e vertida em peneira de 500 "mesh" $(0,025 \mathrm{~mm})$, a fim de eliminar o resíduo do formol, e as amostras foram reduzidas ao volume de $10 \mathrm{~mL}$ de suspensão.

Para quantificação, a suspensão foi manualmente homogeneizada e foi retirada uma alíquota de 1,0 mL, que foi depositada em lâmina de contagem de Peters e observada em microscópio óptico binocular (100x), para quantificação dos espécimes presentes.

O número médio de nematoides extraídos das raízes foi utilizado para cálculo da estimativa do número de nematoides por grama de raiz $(\mathrm{NGR}=$ número de nematoides extraído da raiz/peso do sistema radicular). $\mathrm{O}$ fator de reprodução (FR) foi obtido pela relação população final/população inicial, sendo a população final o número total de nematoides extraídos das raízes de cada planta ao final do experimento, e a população inicial, o número de nematoides usados como inóculo em cada planta. Valores de fator de reprodução menores ou iguais a 1,0 caracterizam reação de resistência, enquanto maiores que 1,0 caracterizam reação de suscetibilidade (Trudgill, 1991).

Para a análise estatística dos resultados, efetuou-se a verificação da homogeneidade da variância, utilizandose o teste de normalidade de Shapiro Wilk, que indicou a necessidade de transformação, a fim de obter-se a estabilização das variâncias. Os dados referentes às variáveis de FR e NGR foram, então, transformados em $\log$ de $x$ e $(x+0,5)^{0,5}$, respectivamente, sendo $x$ o valor original da variável. Posteriormente, os valores de FR e NGR foram submetidos à análise de variância, com auxílio do aplicativo computacional Sisvar (Ferreira, 2011). As médias dos tratamentos foram comparadas pelo teste de Duncan $(\mathrm{p}=0,05)$.

\section{Resultados e Discussão}

Os resultados obtidos evidenciaram que as cultivares de sorgo sacarino testadas ('BRS 506', 'BRS 508', 'BRS 509' e 'BRS 511') foram igualmente ( $\mathrm{p}>0,05)$ resistentes $(\mathrm{FR} \leq 1,0)$ a $M$. javanica (Tabela 1$)$ e a $R$. reniformis (Tabela 2), e suscetíveis (FR > 1,0) a $P$. brachyurus (Tabela 3 ).

A cultivar de soja 'BRS 360RR' e a cultivar de algodão 'BRS Cedro' mostraram ser suscetíveis a, respectivamente, $M$. javanica $(\mathrm{FR}=6,2$ e $\mathrm{NGR}=$ $4665,2)$ e $R$. reniformis $(\mathrm{FR}=4,87$ e $\mathrm{NGR}=213,4)$. A multiplicação de $P$. brachyurus foi relativamente baixa em milho 'BRS 1010' (FR = 2,1 e NGR =6,4), mas ainda assim suficiente para caracterizar a cultivar como suscetível (FR > 1,0). Crotalaria spectabilis apresentou valores de FR próximos a $0,0(0,1 ; 0,3$ e 0,2$)$ nos experimentos com $M$. javanica, $R$. reniformis e $P$. brachyurus, confirmando ser resistente a essas espécies de nematoides (Asmus e Ferraz, 1988; Silva et al., 1989a; Silva et al., 1989b).

Tabela 1. Fator de reprodução (FR) e número de nematoides da espécie $M$. javanica por grama de raiz (NGR) em cultivares de sorgo sacarino, soja e crotalária. Dourados, MS, 2016.

\begin{tabular}{lcc}
\hline TRATAMENTOS & FR $^{1}$ & NGR $^{1}$ \\
\hline SORGO BRS 506 & $0,5 \mathrm{~b}^{2}$ & $35,1 \mathrm{~b}$ \\
SORGO BRS 508 & $0,2 \mathrm{~b}$ & $22,1 \mathrm{~b}$ \\
SORGO BRS 509 & $0,6 \mathrm{~b}$ & $30,8 \mathrm{~b}$ \\
SORGO BRS 511 & $0,3 \mathrm{~b}$ & $21,1 \mathrm{~b}$ \\
SOJA BRS 360 RR & $6,2 \mathrm{a}$ & $4655,2 \mathrm{a}$ \\
CROTALÁRIA & $0,1 \mathrm{~b}$ & $14,2 \mathrm{~b}$ \\
\hline C.V $(\%)$ & 43,85 & 21,13
\end{tabular}

\footnotetext{
${ }^{1}$ Dados originais, médias de oito repetições; ${ }^{2}$ valores seguidos da mesma letra, na coluna, não diferem entre si pelo teste de Duncan (p >0,05).
} 
Tabela 2. Fator de reprodução (FR) e número de nematoides da espécie Rotylenchulus reniformis por grama de raiz (NGR) em cultivares de sorgo sacarino, algodão e crotalária. Dourados, MS, 2016.

\begin{tabular}{lcc}
\hline TRATAMENTOS & FR $^{1}$ & NGR $^{1}$ \\
\hline SORGO BRS 506 & $0,04 \mathrm{~b}^{2}$ & $0,1 \mathrm{~b}$ \\
SORGO BRS 508 & $0,04 \mathrm{~b}$ & $0,8 \mathrm{~b}$ \\
SORGO BRS 509 & $0,03 \mathrm{~b}$ & $0,2 \mathrm{~b}$ \\
SORGO BRS 511 & $0,03 \mathrm{~b}$ & $0,2 \mathrm{~b}$ \\
ALGODÃO BRS CEDRO & $4,87 \mathrm{a}$ & $213,4 \mathrm{a}$ \\
CROTALÁRIA & $0,30 \mathrm{~b}$ & $6,1 \mathrm{~b}$ \\
\hline C.V $(\%)$ & 32,21 & 34,21 \\
\hline
\end{tabular}

${ }^{1}$ Dados originais, médias de oito repetições; ${ }^{2}$ valores seguidos da mesma letra, na coluna, não diferem entre si pelo teste de Duncan (p >0,05).

Tabela 3. Fator de reprodução (FR) e número de nematoides da espécie Pratylenchus brachyurus por grama de raiz (NGR) em cultivares de sorgo sacarino, milho e crotalária. Dourados, MS, 2016.

\begin{tabular}{lcc}
\hline TRATAMENTOS* & FR $^{\mathbf{1}}$ & NGR $^{\mathbf{1}}$ \\
\hline SORGO BRS 506 & $1,6 \mathrm{~b}^{\mathbf{2}}$ & $2,3 \mathrm{~b}$ \\
SORGO BRS 508 & $1,4 \mathrm{~b}$ & $6,5 \mathrm{a}$ \\
SORGO BRS 509 & $2,0 \mathrm{~b}$ & $2,7 \mathrm{~b}$ \\
SORGO BRS 511 & $1,5 \mathrm{~b}$ & $3,0 \mathrm{~b}$ \\
MILHO BRS 1010 & $2,1 \mathrm{a}$ & $6,4 \mathrm{a}$ \\
CROTALÁRIA & $0,2 \mathrm{c}$ & $2,1 \mathrm{~b}$ \\
\hline C.V (\%) & 23,70 & 39,68 \\
\hline I'Dado oring
\end{tabular}

Das cultivares avaliadas no presente experimento, 'BRS 506' já havia sido testada quanto à reação a $M$. javanica por Inomoto et al. (2008), que observaram baixo FR do nematoide, reforçando o potencial da cultivar para uso em áreas infestadas com esta espécie do nematoide das galhas.

Embora sejam escassas as informações sobre as cultivares de sorgo sacarino estudadas neste experimento, há evidências das distintas respostas das cultivares de sorgo granífero e forrageiro, à M. javanica. Nos Estados Unidos, o sorgo granífero é utilizado para rotação com soja em áreas infestadas com nematoides das galhas, pois vários genótipos são considerados maus hospedeiros de $M$. arenaria, $M$. incognita e $M$. javanica (Rich e Kinloch, 2007). Entretanto, o mesmo não ocorre com o sorgo forrageiro, já que estudos realizados no Brasil por Inomoto et al. (2005) em casa de vegetação evidenciaram a suscetibilidade da cultivar 'IPA 7301011', que propiciou aumentos populacionais expressivos, com fatores de reprodução (FR) entre 27,81 e 39,57 para populações de $M$. javanica de Mato Grosso do Sul e São Paulo. Logo, há indícios de que os sorgos granífero, sacarino e forrageiro e o sorgo silageiro, embora pertencentes à mesma espécie botânica, apresentem reações distintas a $M$. javanica.

Os resultados obtidos neste trabalho, embora com cultivares diferentes, reforçam os dados de Carneiro et al. (2007) que, ao estudarem o fator de reprodução de $M$. javanica em sete cultivares de sorgo, concluíram que os híbridos 'BRS 304' (FR=0,7), 'Zeneca 732' (FR= $0,5)$ e 'Planta Baixa $(F R=0,3)$ ' comportam-se como resistentes ao nematoide.

No que diz respeito a $R$. reniformis, os resultados obtidos no presente trabalho indicam que as cultivares de sorgo sacarino avaliadas apresentam uma alta resistência ao nematoide, com valores muito baixos, tanto do fator de reprodução quanto do número de nematoides por grama de raiz, no que foram semelhantes ao padrão de resistência $C$. spectabilis. Em experimentos conduzidos em casa de vegetação, com metodologia semelhante à utilizada no presente trabalho, Asmus (2005) avaliou as cultivares de sorgo "Santa Elisa", "IPA 7301011", "BR 800”, "BR 601", "BR 700" e "BRS 701", concluindo serem todas resistentes a $R$. reniformis e, desta forma, adequadas para cultivo em áreas infestadas por este nematoide. Resultados semelhantes foram obtidos por Asmus et al. (2008) com as cultivares "IPA 7301011" e "Santa Elisa", esta última também avaliada em condições de campo naturalmente infestado, como cultura de cobertura em sucessão ao algodoeiro, resultando na redução da população do nematoide no solo e aumento de produtividade do algodoeiro cultivado em sequência. 
Ao avaliarem a eficiência de seis adubos verdes de inverno e treze de verão sobre a população de $R$. reniformis em solo naturalmente infestado, Gardiano et al. (2014) observaram que o cultivo de sorgo da cultivar 'SI03204' propiciou a redução da população do nematoide no solo $(\mathrm{FR}=0,73)$. Embora com base em número reduzido de experimentos, os resultados de reação de sorgo a $R$. reniformis, incluindo-se as cultivares sacarinas, sugerem que a cultura seja má hospedeira deste nematoide, constituindo-se em boa opção para uso em programas de rotação ou sucessão de culturas em áreas infestadas.

Diferentemente do observado com $M$. javanica e $R$. reniformis, as quatro cultivares de sorgo sacarino avaliadas mostraram-se suscetíveis a $P$. brachyurus, com fatores de reprodução (FR) que variaram de 1,4 a 2,0 e NGR de 2,26 a 6,45 (Tabela 3). Estes resultados se alinham com o trabalho de Sharma e Medeiros (1982), que testaram 16 genótipos de sorgo silageiro (chamado no trabalho de sacarino) em casa de vegetação, e observaram que, 45 dias após a inoculação com 67 espécimes de $P$. brachyurus por planta, as cultivares 'BR 500', 'BR 501', 'BR 502', 'BR 503', 'BR 601', 'BRS 602' e 'SART', e os híbridos intraespecíficos 'CMS $x$ S 516', 'CMS $x$ S603', 'CMS $x$ S623', 'CMS $x$ $\mathrm{S} 717$ ', 'CMS $x$ S 719', 'CMS $x$ S 723', 'CMS $x$ S733', 'CMS $x$ S634' e 'CMS x S 735' apresentaram FR variável entre 7,2 e 26,17 . Em experimento semelhante, mas com 90 dias de duração, Charchar e Huang (1980) verificaram aumento populacional (FR) de 1,96 vezes em sorgo 'MS399'. Uma exceção à suscetibilidade de $S$. bicolor a $P$. brachyurus é encontrada no trabalho de Endo (1959), que verificou redução populacional (FR = 0,12) na cultivar 'Common'. No entanto, no conjunto dos resultados disponíveis corroborados pelo presente trabalho, evidencia-se a afirmação de Inomoto (2008), o qual relata que tanto o sorgo sacarino como o forrageiro (S. bicolor x S. sudanense) são bons hospedeiros dessa espécie de nematoide.

\section{Conclusões}

Nas condições em que foram realizados os experimentos, as cultivares de sorgo sacarino 'BRS 506', 'BRS 508', 'BRS 509' e 'BRS 511' são resistentes a $M$. javanica e $R$. reniformis, e suscetíveis a P. brachyurus.

\section{Agradecimentos}

À Coordenação de Aperfeiçoamento de Pessoal de Nível Superior (CAPES), pela concessão de bolsa de estudos à primeira autora.

\section{Referências Bibliográficas}

Asmus, G. L., Inomoto, M. M., Cargini, R. A. 2008. Culturas de cobertura para o manejo do nematoide reniforme em algodoeiro: avaliações em casa de vegetação e campo. Tropical Plant Pathology, 33, 85-89.

Asmus, G. L., Richetti, A. 2010. Rotação de culturas para o manejo do nematoide reniforme em algodoeiro. DouradosMS: Embrapa Agropecuária Oeste, 26 p. (Boletim de Pesquisa e Desenvolvimento, n. 55).

Asmus, G. L. 2005. Reação de algumas culturas de cobertura utilizadas no sistema plantio direto ao nematoide reniforme. Embrapa Agropecuária Oeste, Dourados, 4p. (Embrapa Agropecuária Oeste. Comunicado técnico, 99).

Asmus, R. M. F., Ferraz, S. 1988. Antagonismo de algumas espécies vegetais, principalmente leguminosas, a Meloidogyne javanica. Fitopatologia Brasileira, 13, 20-24.

Carneiro, R., Moritz, M. P., Mônaco, A. P. A., Nakamura, K. C., Scherer, A. 2007. Reação de milho, sorgo e milheto a Meloidogyne incognita, M. javanica e M. paranaensis. Nematologia Brasileira, 31, 9-13.

Charchar, J. M., Huang, C. S. 1980. Círculo de hospedeiras de Pratylenchus brachyurus. I - Graminae. Fitopatologia Brasileira, 5, 351-357.

Coolen, W. A., D'Herde, C. J. A. 1972. Method for the quantitative extraction of nematodes from plant tissue. State Nematology and Entomology Research Station, Ghent, 77 p.

Dias, W. P., Asmus, G. L., Silva, J. F. V., Garcia, A., Carneiro, G. E. S. 2010. Nematóides, in: Almeida, A. M. R., Seixas, C. D. S. (Ed.) Soja: doenças radiculares e de hastes e inter-relações como manejo do solo e da cultura. Embrapa Soja, Londrina, pp. 173-206.

Dinardo-Miranda, L. L., Gil, M. A., Menegatti, C. C. 2003. Danos causados por nematoides a variedades de cana-deaçúcar em cana planta. Nematologia Brasileira, 27, 69-73.

Endo, B. Y. 1959. Responses of root-lesion nematodes, Pratylenchus brachyurus and P. zeae, to various plants and soil types. Phytopathology, 49, 417-421.

Ferreira, D. F. 2011. Sisvar: a computer statistical analysis system. Ciência e Agrotecnologia, 35, 1039-1042.

Galbieri, R., Inomoto, M. M., Silva, R. A.; Asmus, G. L. 2012. Os nematoides na cultura do algodoeiro em Mato Grosso, in: Belot, J., (Org.) Manual de boas práticas de manejo do algodoeiro em Mato Grosso. IMA MT - AMPA, Cuiabá, pp. 150-161.

Gardiano, C. G., Krzyzanowski, A. A., Saab, O. J. G. 2014. Eficiência de espécies de adubos verdes sobre a população do nematoide reniforme. Semina: Ciências Agrárias, 35, 719-726.

Gonzaga, V., Santos, J. M., Mendonça, R. S., Santos, M. A. 2016. Gênero Pratylenchus, in: Oliveira, C. M. G., Santos, M. A., Castro, L. H. S. (Org.) Diagnose de Fitonematoides. Millennium, Campinas, pp. 71-98. 
Inomoto, M. M. 2011. Avaliação da resistência de 12 híbridos de milho a Pratylenchus brachyurus. Tropical Plant Pathology, 36, 308312 .

Inomoto, M. M., Antedomênico, S. R., Santos, V. P., Silva, R. A., Almeida, G. C. 2008. Avaliação em casa de vegetação do uso de sorgo, milheto e crotalária no manejo de Meloidogyne javanica. Tropical Plant Pathology, 33, 125-129.

Inomoto, M. M., Asmus, G. L., Ferraz, M. A., Sazaki, C. S. S., Schirmann, M.R. 2005. Reação de dez coberturas vegetais utilizadas no sistema plantio direto a Meloidogyne javanica. Summa Phytopathologica, 31, 367-370.

Inomoto, M. M., Motta, L. C. C., Machado, A. C. Z., Sazaki, C. S. S. 2006. Reação de dez coberturas vegetais a Pratylenchus brachyurus. Nematologia Brasileira, 30, 151-157.

Lordello, L.G.E. 1981. Nematóides das Plantas Cultivadas. 6 ed. Nobel, São Paulo.

May, A., Souza, V. F., Silva, A. F., Teixeira, M. F. F. 2015. Comportamento produtivo de sorgo sacarino em função do arranjo de plantas. Embrapa Milho e Sorgo, Sete Lagoas (Boletim de Pesquisa e Desenvolvimento, 116), $27 \mathrm{p}$.

McClure, M., Husman, S., Schmitt, M. 1999. Infection of sorghum varieties by the Cotton Root-knot Nematode, Meloidogyne incognita. University of Arizona, Tucson (Cotton Report), 610 p.

McSorley, R. 1998. Population Dynamics, in: Barker, K.R., Pederson, G. A., Windhan, G.L. (Eds.), Plant and Nematode Interactions. American Society of Agronomy, Madison, pp. 109-133.

Miranda, R. A. 2012. Aspectos econômicos da produção de etanol a partir do sorgo sacarino plantado na entressafra da cana-de-açúcar, in: May, A., Mantovani, E. C., Durães, F. O. M., Vasconcellos, J. H., Parrella, R. A. C., Schaffert, R. E., Parentoni, S. N. (Eds.), Seminário Temático Agroindustrial de Produção de Sorgo Sacarino para Bioetanol - Anais. Embrapa Milho e Sorgo, Sete Lagoas-MG (Documentos 145), pp. 59-62.
Motalaote, B., Starr, J. L., Frederiksen, R. A., Miller, F. R. 1987. Host status and susceptibility of sorghum to Pratylenchus species. Revieu de Nematologie, 10, 81-86.

Rich, J. R., Kinlonch, R. A. 2007. Sorghum nematode management. http://www.edis.ifas.ufl.edu/NG017 (acessado 10 novembro 2014).

Robinson, A. F., Inserra, R. N., Caswell-Chen, E. P., Vovlas, N., Troccoli, A. 1997. Rotylenchulus species: identification, distribution, host ranges, and crop plant resistance. Nematropica, 27, 127-180.

Sharma, R. D., Medeiros, A. C. S. 1982. Reações de alguns genótipos de sorgo sacarino aos nematoides Meloidogyne javanica e Pratylenchus brachyurus. Pesquisa Agropecuária Brasileira, 17, 697-701.

Silva, G. S., Ferraz, S., Santos, J. M. 1989a. Resistência de espécies de Crotalaria a Pratylenchus brachyurus e P. zeae. Nematologia Brasileira, 13, 81-86.

Silva, G. S.; Ferraz, S., Santos, J. M. 1989b. Resistência de espécies de Crotalaria a Rotylenchulus reniformis. Nematologia Brasileira, 13, 87-92.

Spaull, V. W., Cadet, P. 1990. Nematode Parasites of Sugarcane, in: Luc, M., Sikora, R. A., Bridge, J., (Eds) Nematodes on Tropical and Sub-Tropical Agriculture. CAB International, Oxon, pp. 461-491.

Taylor, A. L., Sasser, J. N. 1978. Biology, identification and control of root-knot nematodes (Meloidogne spp.). Department of Plant Pathology, North Carolina State University Graphics, Raleigh, 111p.

Trudgill, D. L. 1991. Resistance to and tolerance of plant parasitic nematodes in plants. Annual Review of Phytopathology, 29, p.167-192. 